\title{
Review Article \\ Preoperative Chemotherapy for Gastric Cancer: Personal Interventions and Precision Medicine
}

\author{
Wei Xu, Maneesh K. Beeharry, Wentao Liu, Min Yan, and Zhenggang Zhu \\ Shanghai Key Laboratory of Gastric Neoplasms, Shanghai Institute of Digestive Surgery, Department of Surgery, \\ Ruijin Hospital, Shanghai Jiao Tong University, Shanghai 200025, China \\ Correspondence should be addressed to Wentao Liu; wt_mygod@163.com
}

Received 28 July 2016; Accepted 4 December 2016

Academic Editor: Mario Scartozzi

Copyright (C) 2016 Wei Xu et al. This is an open access article distributed under the Creative Commons Attribution License, which permits unrestricted use, distribution, and reproduction in any medium, provided the original work is properly cited.

\begin{abstract}
In spite of the declining incidence of gastric cancer (GC) in recent years, the mortality rate is still high. The asymptomatic nature and nonspecific clinical manifestations combined with the lack of efficient screening programs delay the diagnosis of GC. Therefore, the prevalence of advanced gastric cancer (AGC) has prompted the need for aggressive and intensive treatment options. Among the various treatment options for AGC, surgery is still the mainstay. However, the efficacy of surgery alone is not established. Results from multiple randomized controlled trials suggest that preoperative chemotherapy is promising intervention for the treatment and management of AGC. The main objective of neoadjuvant chemotherapy is to downstage or control micrometastasis in resectable tumor before surgery. On the other hand, conversion chemotherapy refers to surgical treatment aiming at R0 resection after chemotherapy for originally nonresectable or marginally resectable tumors. Nevertheless, preoperative chemoradiotherapy is considered beneficial for AGC patients. Over the last few decades, the combination of chemotherapy and targeted therapy prior to surgery demonstrated great results for the treatment of AGC. The rapid developments in genomics and proteomics have heralded the era of precision medicine. The combination of preoperative chemotherapy and precision medicine may enhance survival in AGC patients.
\end{abstract}

\section{Introduction}

Gastric cancer (GC) is the fifth most common malignancy and the third leading cause of cancer death globally [1]. More than $70 \%$ of GC cases occur in developing countries and Eastern Asia accounts for half of the total incidence, with the highest estimated mortality rate [1]. The 5 -year overall survival rate in patients with resectable GC is only about $20 \%$ to $30 \%$ worldwide. However, the overall 5-year survival rate of GC patients in Japan is about 70\%, which is higher than in other countries $[2,3]$. Surgery is the cornerstone of treatment for GC. However, the prognosis of patients with locally advanced GC is still poor even after curative resection [4]. In order to improve the prognosis of patients with advanced GC (AGC), a multimodal strategy is desirable. Preoperative chemotherapy has potential benefits such as downstaging the primary tumor to increase the likelihood and efficacy of curative resection, while simultaneously eliminating possible micrometastases to prevent or reduce tumor recurrence and metastasis, thus improving and managing tumor-associated symptoms [5]. The MAGIC trial was the first large randomized controlled trial to demonstrate the effect of perioperative chemotherapy in GC. Perioperative chemotherapy decreases tumor size and tumor stage, leading to improved progressionfree and overall survival rates in patients with resectable GC [5]. However, not all AGC patients benefit from preoperative chemotherapy: studies suggest that nearly $15 \%$ of patients undergoing preoperative chemotherapy show risks of tumor progression [6,7]. Therefore, predicting patients' response to preoperative chemotherapy remains particularly crucial. Advances in modern medicine paved the way for precision medicine. Precision medicine is a new medical concept and model that has blossomed in USA. It is based on individual medicine, rapid progress in genome sequencing technology, and innovations resulting from cross-pollination between biological information and big-data science [8]. Different patients show different tolerance to chemotherapy and manifest varying degrees of curative response. Advances 
TABle 1: Preoperative and perioperative chemotherapy in resectable GC.

\begin{tabular}{|c|c|c|c|c|c|}
\hline Reference & Selection criteria & $\begin{array}{l}\text { Experimental versus control } \\
\text { group }\end{array}$ & $\begin{array}{l}\text { Patients } \\
\quad(n)\end{array}$ & $\begin{array}{l}\mathrm{R} 0 \text { resection } \\
(\%)\end{array}$ & $\begin{array}{l}\text { Survival rate } \\
(\%)\end{array}$ \\
\hline $\begin{array}{l}\text { Songun et al. [9] } \\
\text { and Hartgrink } \\
\text { et al. [10] }\end{array}$ & $\begin{array}{l}\text { T2-T4; } \\
\text { M0; GC }\end{array}$ & $\begin{array}{c}\text { FAMTX } \times 2-4+\text { surgery versus } \\
\text { surgery alone }\end{array}$ & 27 versus 29 & 56 versus 62 & $\begin{array}{l}32 \text { versus } 53 \\
\text { (5 years) }\end{array}$ \\
\hline $\begin{array}{l}\text { Cunningham et } \\
\text { al. [5] }\end{array}$ & $\begin{array}{l}\text { Resectable GC, GEJ, or lower } \\
\text { esophagus }\end{array}$ & $\begin{array}{c}\mathrm{ECF} \times 3+\text { surgery }+\mathrm{ECF} \times 3 \\
\text { versus surgery alone }\end{array}$ & $\begin{array}{l}250 \text { versus } \\
253\end{array}$ & 74 versus 68 & $\begin{array}{l}36 \text { versus } 23 \\
\text { (5 years) }\end{array}$ \\
\hline Ychou et al. [11] & $\begin{array}{l}\text { Resectable GC, GEJ, or lower } \\
\text { esophagus }\end{array}$ & $\begin{array}{c}\mathrm{FP} \times 2-3+\text { surgery }+\mathrm{FP} \times 3-4 \\
\text { versus surgery alone }\end{array}$ & 113 versus 111 & 84 versus 73 & $\begin{array}{l}38 \text { versus } 24 \\
\text { ( } 5 \text { years })\end{array}$ \\
\hline $\begin{array}{l}\text { Schuhmacher et } \\
\text { al. [12] }\end{array}$ & Locally advanced GC; GEJ & $\begin{array}{c}\mathrm{PFL} \times 2+\text { surgery versus surgery } \\
\text { alone }\end{array}$ & 72 versus 72 & $\begin{array}{l}81.9 \text { versus } \\
66.7\end{array}$ & $\begin{array}{l}72.7 \text { versus } \\
69.9(2 \text { years })\end{array}$ \\
\hline Oyama et al. [13] & $\begin{array}{c}\text { Advanced GC with PAN } \\
\text { metastasis }\end{array}$ & $\begin{array}{c}\text { DCS + surgery versus surgery } \\
\text { alone }\end{array}$ & 16 versus 28 & NS & $\begin{array}{c}93.8 \text { versus } \\
32.9 \text { ( } 2 \text { years })\end{array}$ \\
\hline $\begin{array}{l}\text { Kinoshita et al. } \\
{[14]}\end{array}$ & $\begin{array}{l}\text { Potentially resectable stage IV } \\
\text { GC }\end{array}$ & DCS + surgery versus DCS & 34 versus 23 & NS & $\begin{array}{c}50.1 \text { versus } \\
0.0 \text { (3 years) }\end{array}$ \\
\hline
\end{tabular}

GC: gastric cancer; GEJ: gastroesophageal junction; FAMTX: 5-fluorouracil, doxorubicin, and methotrexate; ECF: epirubicin, cisplatin, and fluorouracil; FP: fluorouracil and cisplatin; PFL: cisplatin, fluorouracil, and d-L-folinic acid; PAN: para-aortic lymph node; DCS: docetaxel, cisplatin, and S-1; NS: not stated.

in genome technology facilitate the design of individual chemotherapy regimens with the most promising curative outcome and the least number of adverse effects. Thus, patients benefit from individualized treatment plans tailored to their genetic constitution. The evolution and design of precision interventions for GC patients are clinically significant. The combination of preoperative chemotherapy with precision medicine enhances the therapeutic outcomes.

\section{Preoperative and Perioperative Chemotherapy with Prognosis}

The Dutch FAMTX trial was the first randomized controlled trial to investigate the role of preoperative chemotherapy in resectable GC (Table 1). Patients with early GC were excluded. The trial compared the outcomes of patients undergoing surgery with and without preoperative chemotherapy using the FAMTX regimen. Four cycles of FAMTX chemotherapy were administered to patients in the preoperative chemotherapy group before surgery. The chemotherapy regimen consisted of 5-fluorouracil, doxorubicin, and methotrexate, which was the gold standard at the time. However, this study failed to demonstrate if FAMTX chemotherapy was appropriate for preoperative treatment of patients with operable GC [9]. After a median follow-up of 83 months, $36 \%$ of patients in the preoperative chemotherapy group showed tumor progression. Further, the rate of curative resection and median survival decreased $[9,10]$. Preoperative FAMTX had no beneficial effect on patients with operable GC. Nevertheless, the MAGIC and the FNCLCC/FFCD trials demonstrated the advantages of perioperative chemotherapy in increasing the $\mathrm{R} 0$ resection rate and improving the overall survival in patients $[5,11]$. Even though the chemotherapy regimens used in the two trials varied, the results were consistent.

The MAGIC randomized trial compared surgery with and without perioperative chemotherapy using ECF regimen. In this trial, patients with resectable adenocarcinoma of the stomach, gastroesophageal junction, or lower esophagus were randomly assigned to surgery alone or surgery combined with perioperative chemotherapy. Three cycles of chemotherapy were administered to patients in the perioperative chemotherapy group before surgery and three additional cycles after the surgery. The chemotherapy regimen consisted of intravenous epirubicin and cisplatin on day 1 with a continuous intravenous infusion of fluorouracil for three weeks. After a median follow-up of four years, the perioperative ECF chemotherapy group manifested decreased tumor size and tumor stage, resulting in improved progressionfree and overall survival rates [5]. However, $8.4 \%$ of patients in the perioperative chemotherapy group failed to complete surgery, and only $69.3 \%$ received curative resection while $34 \%$ did not complete postoperative chemotherapy [5]. Therefore, the patient response to perioperative chemotherapy regimen varied, suggesting the need for specialized and personalized regimens.

Another classic randomized clinical intervention included the FNCLCC and FFCD multicenter phase III trial. The trial conclusions were consistent with the results of the MAGIC trial. In this trial, patients with resectable adenocarcinoma of the stomach, gastroesophageal junction, or the lower esophagus were randomly assigned to surgery alone or perioperative chemotherapy with surgery. Two to three preoperative cycles were administered to the patients in the perioperative chemotherapy group and three to four postoperative cycles of chemotherapy. The chemotherapy regimen combined two drugs and did not include epirubicin, in contrast to the MAGIC trial. The chemotherapy regimen consisted of intravenous cisplatin on day 1 and continuous intravenous infusion of fluorouracil from day 1 to day 5 every four weeks. After a follow-up of five years, this trial also demonstrated that the perioperative FP chemotherapy regimen increased the rates of curative resection, diseasefree survival, and overall survival [11]. However, in the perioperative chemotherapy group, $11 \%$ of the patients showed disease progression after preoperative chemotherapy whereas $3.5 \%$ patients did not undergo surgery and $84 \%$ underwent $\mathrm{R} 0$ resection [11]. The trial also included a few patients 
who failed to derive any survival benefit from preoperative chemotherapy. In the MAGIC and FNCLCC/FFCD trials, the combination of cisplatin and fluorouracil was effective perioperative chemotherapy regimens in AGC patients. However, the value of preoperative chemotherapy alone was unclear.

The European Organization for Research and Treatment of Cancer Randomized Trial 40954 was designed to evaluate the role of preoperative chemotherapy. In this trial, patients with locally advanced adenocarcinoma of the stomach or gastroesophageal junction were randomly assigned to surgery alone and preoperative chemotherapy with surgery. This trial did not include postoperative chemotherapy, in contrast to the MAGIC and FNCLCC/FFCD trials. The patients in the preoperative chemotherapy group were treated with two 48-day cycles. The chemotherapy regimen consisted of intravenous cisplatin on days 1, 15, and 29 and continuous intravenous infusion of d-L-folinic acid and fluorouracil on days $1,8,15,22,29$, and 36. Due to poor accrual, the trial was prematurely terminated after enrolling 144 patients. After a median follow-up of 4.4 years, the trial only demonstrated an increased $\mathrm{R} 0$ resection rate in the preoperative chemotherapy group, without any survival benefit, and unfortunately increased the incidence of postoperative complications [12]. The trial was statistically weak. However, it also suggested that preoperative chemotherapy failed to result in any survival benefit. In brief, the MAGIC, FNCLCC/FFCD, and EORTC 40954 trials demonstrated that preoperative chemotherapy increased the $\mathrm{R} 0$ resection rate. Recent studies demonstrated the effectiveness of preoperative chemotherapy in patients with locally advanced GC.

Nevertheless, preoperative chemotherapy is also indicated for the treatment of AGC with distal metastasis. It is well known that the prognosis of GC patients with para-aortic lymph node (PAN) metastasis is unfavorable. In a study by Oyama et al. [13], the efficacy of preoperative chemotherapy with docetaxel, cisplatin, and S-1 (DCS therapy) and curative resection for GC with pathologically positive PAN was investigated. The findings suggested that preoperative chemotherapy (DCS regimen) followed by gastrectomy was particularly effective and feasible for AGC with PAN metastasis. The 2-year survival rate was $32.9 \%$ in patients without preoperative chemotherapy and $93.8 \%$ in patients with preoperative chemotherapy [13]. Furthermore, Kinoshita et al. [14] investigated the efficacy of conversion gastrectomy following preoperative chemotherapy (DCS regimen) in patients with potentially resectable stage IV GC. Patients who underwent conversion gastrectomy following preoperative DCS therapy showed a 3-year overall survival rate of $50.1 \%$, which was longer than in DCS therapy alone. By contrast, in potential resectable cases, the 3-year overall survival rate was $92.9 \%$. These two trials emphasize the efficacy of preoperative chemotherapy in advanced gastric cancer, suggesting a new direction in the treatment of AGC.

\section{Comparison between Different Chemotherapy Regimens}

With the development of preoperative chemotherapy, several different combinations of regimens are available for the treatment of GC. The regimen consists of either single or multiple drugs administered orally, intravenously, or both.

The MAGIC trial investigated the combination of epirubicin, cisplatin, and 5-FU. This trial revealed the significance of preoperative chemotherapy. In an effort to develop chemotherapy regimens with improved efficacy for AGC patients, Cunningham et al. [15] investigated capecitabine and oxaliplatin as alternatives to fluorouracil and cisplatin infusion, respectively. In their study, 1002 patients were randomly assigned to four different chemotherapy regimens (ECF, ECX, EOF, and EOX). The median survival in the ECF, ECX, EOF, and EOX groups was 9.9, 9.9, 9.3, and 11.2 months, respectively, while the 1-year survival rates were $37.7 \%, 40.8 \%, 40.4 \%$, and $46.8 \%$, respectively [15]. There was no significant difference in the progression-free survival and response rates in the four regimens. Capecitabine and fluorouracil displayed similar toxic effects. When compared with cisplatin, oxaliplatin showed higher incidence of diarrhea and neuropathy and a lower incidence of neutropenia, alopecia, renal toxicity, and thromboembolism [15]. Kang et al. [16] conducted a randomized phase III noninferiority trial comparing capecitabine/cisplatin (XP) with 5fluorouracil/cisplatin (FP) as the first-line therapy in AGC patients. The median progression-free survival was 5.6 versus 5.0 months while the median overall survival was 1.5 versus 9.3 months, respectively [16]. The results suggested that XP was more effective than FP and that capecitabine was better than 5-fluorouracil. The Swiss Group for Clinical Cancer Research conducted a randomized phase II trial, which compared docetaxel-cisplatin-5-FU (DCF) versus docetaxelcisplatin (DC) and ECF. The study concluded that docetaxelbased regimens were more efficacious than ECF, despite being associated with increased toxicity. They suggested that DCF was the more promising regimen and should be selected for formal comparison with ECF [17]. When 5-FU was switched to $S-1$, the efficacy of the combination of docetaxel, cisplatin, and S-1 was remarkable. Hirakawa et al. [18] and Oki et al. [19], respectively, studied the efficacy of preoperative chemotherapy regimens such as DCS and DS. Hirakawa et al. found that DCS preoperative chemotherapy resulted in an $\mathrm{R} 0$ resection rate of $90.7 \%$ and the rate of pathological response was $65.9 \%$ [18]. Oki et al. found that the rate of curative resection was $93.6 \%$ and the pathological response rate was $47 \%$ in the DS preoperative chemotherapy group [19]. The results of the two trials were very similar and suggested that both DCS and DS regimen therapies were indicated as preoperative chemotherapy regimen for patients with resectable AGC.

Nevertheless, preoperative chemotherapy regimen is not a gold standard for locally advanced GC. GC is complex disease refractory to monotherapy or single drug regimens. In general practice, drugs such as capecitabine, S-1, and docetaxel are more frequently used in preoperative chemotherapy. Additional studies investigating these regimens yielded significantly encouraging results. In order to design the best treatment strategy for patients, it is necessary to adopt a multimodal strategy for locally advanced GC cases. Preoperative chemotherapy is an important part of multimodal management. It is essential to focus on the relationship 
TABLE 2: Preoperative chemoradiotherapy.

\begin{tabular}{|c|c|c|c|c|c|c|}
\hline Reference & $\begin{array}{l}\text { Patients } \\
(n)\end{array}$ & treatment & Surgery $(\%)$ & $\begin{array}{l}\text { R0 resection } \\
(\%)\end{array}$ & PCR (\%) & $\begin{array}{l}\text { Survival rate }(\%) \text { or } \\
\text { median survival (mo) }\end{array}$ \\
\hline Lowy et al. [21] & 24 & $5-\mathrm{FU}+45 \mathrm{EBRT}$ & 83 & 75 & 11 & NS \\
\hline $\begin{array}{l}\text { Wydmański et } \\
\text { al. [22] }\end{array}$ & 40 & 5-FU + LV + 45 Gy EBRT & 80 & 75 & 18 & $63 \%$ ( 2 years $)$ \\
\hline Allal et al. [23] & 19 & $5-\mathrm{FU}+\mathrm{LV}+\mathrm{C}+38.4 \mathrm{~Gy}$ & 100 & NS & 5 & $35 \%$ (5 years) \\
\hline Ajani et al. [24] & 34 & 5-FU, LV, C + 45 Gy EBRT, 5-FU & 85 & 70 & 30 & 33.7 months \\
\hline Ajani et al. [25] & 41 & 5-FU, C, P + 45 Gy EBRT, 5-FU, P & 98 & 78 & 20 & NS \\
\hline Ajani et al. [26] & 49 & 5-FU, LV, C + 45 Gy EBRT, 5-FU, P & 83 & 77 & 26 & 23.2 months \\
\hline Stahl et al. [27] & 126 & $\begin{array}{l}\text { 5-FU, LV, C + surgery versus 5-FU, LV, C } \\
\quad+30 \mathrm{~Gy}, \mathrm{C} \text {, etoposide + surgery }\end{array}$ & 88 versus 82 & 69 versus 72 & $\begin{array}{l}2.0 \text { versus } \\
15.6\end{array}$ & $\begin{array}{c}27.7 \text { versus } 47.4(3 \\
\text { years })\end{array}$ \\
\hline Inoue et al. [29] & 12 & S-1, 50 Gy EBRT & 100 & 92 & 17 & 58.3 (3 years) \\
\hline Lee et al. [30] & 12 & S-1,oxaliplaitin, 41.4 Gy EBRT & 100 & 92 & 8 & NS \\
\hline Trip et al. [31] & 25 & Carboplatin, P + 45 Gy EBRT & 96 & 72 & 16 & NS \\
\hline $\begin{array}{l}\text { van Hagen et al. } \\
{[32]}\end{array}$ & 366 & $\begin{array}{l}\text { Carboplatin, P, } 41.4 \text { Gy EBRT + surgery } \\
\text { versus surgery alone }\end{array}$ & 94 versus 99 & 92 versus 69 & 29 versus 49 & $\begin{array}{c}49,4 \text { mos versus } 24.0 \\
\text { mos }\end{array}$ \\
\hline
\end{tabular}

PCR: pathologic complete response; EBRT: external beam radiotherapy; NS: not stated; 5-FU: fluorouracil; LV: leucovorin; C: cisplatin; P: paclitaxel.

between the different treatment regimens and the patients to determine the regimen that is most appropriate, in terms of efficacy and safety. Such an approach will catapult preoperative chemotherapy for GC from an individualized treatment to precision medicine.

\section{Perioperative Chemoradiotherapy}

The intergroup 0116 trial [20] demonstrated the efficacy of postoperative chemoradiotherapy leading to the use of preoperative chemoradiotherapy (CRT) in GC. Recent studies demonstrated that preoperative CRT improved the rate of R0 resection and pathological response without increasing the morbidity or mortality (Table 2). In the studies investigating preoperative CRT, different preoperative chemotherapy regimens were used resulting in different outcomes in patients. The regimens consisted of one, two, three, or even four drugs. Lowy et al. [21] published a pilot study of preoperative chemoradiotherapy for resectable GC in 2001 involving subjects treated with $45 \mathrm{~Gy}$ of external beam radiotherapy (EBRT) with concurrent 5-FU followed by surgery and $10 \mathrm{~Gy}$ intraoperative radiotherapy. The results demonstrated that preoperative CRT was safe. The rates of R0 resection and complete pathologic response were $75 \%$ and $11 \%$, respectively. In addition to 5-FU, the combination of 5-FU and leucovorin was used in preoperative chemoradiotherapy for patients with operable GC. Wydmański et al. [22] conducted a study of preoperative CRT with a concurrent chemotherapy regimen of 5-FU combined with leucovorin. The results suggested that the rates of $\mathrm{R} 0$ resection and complete pathologic response were $75 \%$ and $18 \%$, respectively. The addition of leucovorin to 5-FU slightly increased the complete pathologic response rate but not the R0 resection. Furthermore, many trials investigated the outcome of combining cisplatin, paclitaxel, or both with 5-FU and leucovorin. Allal et al. [23] and Ajani et al. [24] investigated the preoperative CRT using the combination regimen of 5-FU, leucovorin, and cisplatin and demonstrated the safety of preoperative CRT. However, the rate of pathologic complete response (PCR) was significantly different (5\% versus $30 \%)$. The number of patients in the Allal et al. study was limited since there were only 19 patients included in this trial compared with the 34 patients in Ajani et al. study. Therefore, in order to further investigate the combination of 5-FU, leucovorin, and cisplatin with perioperative CRT, larger randomized controlled trialsare is needed. Ajani et al. [24-26] conducted several trials with different chemotherapy regimens in preoperative CRT and the results were consistent with those of the previous study. In Germany, Stahl et al. [27] conducted a phase III randomized controlled trial, designed to compare the value of preoperative chemotherapy with preoperative chemoradiotherapy in patients with locally advanced adenocarcinoma of the gastroesophageal junction but not distal GC. A total of 126 patients were randomly assigned to two groups: Group A included preoperative chemotherapy and Group B represented preoperative chemoradiotherapy. Group A included patients treated with induction chemotherapy with 2.5 cycles of fluorouracil, leucovorin, and cisplatin followed by surgery while Group B treatment consisted of 2 cycles of chemotherapy of fluorouracil, leucovorin, and cisplatin and 3 weeks of combined chemoradiotherapy with cisplatin and etoposide followed by surgery. This trial was prematurely stopped because of the low accrual. However, the results suggested that preoperative CRT for gastroesophageal adenocarcinoma increased the pathologic response and improved the 3-year survival rate significantly [27]. In all of the above-mentioned cases, the preoperative CRT consisted of 5-FU. It is known that $\mathrm{S}-1$ is an oral product of $5-\mathrm{FU}$, which is highly effective and safe in GC therapy [28]. Inoue et al. [29] and Lee et al. [30] explored the feasibility and safety of preoperative CRT with S-1 for the treatment of locally advanced GC. In the Lee et al. study, the concurrent chemotherapy also included oxaliplatin. The two trials demonstrated that S-1 was safe and effective in preoperative CRT. We believe that not only 
S-1 monotherapy but also the combination of S-1 with other drugs should be investigated in preoperative CRT for patients with locally advanced GC.

Recently, a new regimen for preoperative CRT has been reported. Trip et al. [31] and van Hagen et al. [32] investigated the combination of paclitaxel and carboplatin and their results were consistent and promising. In Netherlands, van Hagen et al. [32] conducted a phase III randomized controlled trial to investigate the role of preoperative chemoradiotherapy in the treatment of patients with esophageal or gastroesophageal junction cancer. A total of 366 patients were randomly assigned to surgery alone or chemoradiotherapy (carboplatin, paclitaxel, and $41.4 \mathrm{~Gy}$ in 23 fractions) followed by surgery. Among the patients, $75 \%$ had adenocarcinoma, $23 \%$ had squamous-cell carcinoma, and $2 \%$ had large-cell undifferentiated carcinoma. RO resection was achieved in $92 \%$ of patients in the chemoradiotherapy-surgery group compared with only $69 \%$ of patients in surgery alone group. Further, 29\% of patients in the chemoradiotherapy-surgery group manifested a complete pathological response. The median overall survival was 49.9 months in patients undergoing chemoradiotherapy followed by surgery and 24 months in patients undergoing surgery alone. Although several international trials suggested that preoperative CRT in GC improved $\mathrm{R} 0$ resection and pathological complete response rate, randomized controlled trials for GC are still scarce. The two randomized controlled trials by Stahl et al. and van Hagen et al. were encouraging and promising. There is a need for randomized controlled trial investigating chemoradiotherapy in distal GC to validate the use of chemoradiotherapy before surgery.

\section{Preoperative Chemotherapy Combined with Targeted Therapy}

In light of the rapid developments in the field of tumor biology, targeted therapy has garnered increased attention. Targeted therapy shows great promise for the treatment of AGC patients. The combination of chemotherapy with targeted therapy not only improved the PCR and overall survival but also reduced nonselective toxicity and resistance. Several signaling pathways mediate GC, such as ErbB, VEGF, $\mathrm{PI} 3 \mathrm{~K} / \mathrm{AKT} / \mathrm{mTOR}$, and HGF/MET [50]. The family of ErbB consists of four members, such as epidermal growth factor receptor (EGFR) and the EGFR-related receptors: HER2, HER3, and HER4 [51]. HER2 has been one of the hottest centers of research in the field of gastrointestinal cancer in recent years. A few clinical trials were designed to explore the efficacy of anti-HER2 monoclonal antibodies. Trastuzumab is a humanized recombinant monoclonal antibody that selectively inhibits HER2. ToGA is a phase III, open-labeled randomized controlled trial comparing trastuzumab combined with chemotherapy versus chemotherapy alone for patients with HER2-positive AGC or GJC. The selected chemotherapy regimens were cisplatin plus capecitabine or fluorouracil plus cisplatin. The median overall survival was 13.8 months in the chemotherapy plus trastuzumab group and 11.1 months in the chemotherapy alone group. This trial concluded that the combination of trastuzumab with chemotherapy represented a new standard treatment option for patients with HER2positive AGC or GJC [52]. The Japan Clinical Oncology Group study JCOG1301 (Trigger Study) underway in Japan is a phase II study evaluating the efficacy of SP (S-1 and cisplatin) combined with trastuzumab compared with PS alone. It resulted in favorable outcome in patients with HER2positive AGC and GJC diagnosed with extensive lymph node metastasis [53]. Nevertheless, the benefit of preoperative chemotherapy combined with targeted therapy has not been extensively investigated.

\section{Preoperative Chemotherapy and Precision Medicine}

Precision medicine is a new medical concept and model developed in the West. This concept includes precision diagnosis, precision treatment, and precision prognosis. The diagnosis of GC is not difficult but challenging for precise treatment and accurate prognosis. Therefore, refining the preoperative chemotherapy is the focus of several studies. Notwithstanding the advances in the fields of genomics and proteomics, genetic markers and proteins facilitate the prediction and screening of patients' response to preoperative chemotherapy. Precision medicine ensures that the patients are treated cost-effectively with the most appropriate therapy.

5 -FU and cisplatin are routinely used for preoperative chemotherapy of AGC. Napieralski et al. [33] evaluated the expression of seven therapy-related genes to predict the clinical outcome of patients with AGC treated with preoperative chemotherapy. A total of 61 patients who received 5-FU and cisplatin-based preoperative chemotherapy were followed up. The expressions of 5-FU-related genes including thymidylate synthase (TS), dihydropyrimidine dehydrogenase $(D P D)$, and thymidine phosphorylase $(T P)$ and cisplatin-related genes including excision repair cross complementing 1 (ERCC1), ERCC4, KU80 (an enzyme involved in nonhomologous end joining repair) and growth arrest and DNA-damage-inducible protein 45 alpha (GADD45A) were analyzed using quantitative real-time PCR. The TS expression was not associated with the response. However, patients with a TS expression value $\leq 344.19 \times 10^{-3}$ in the tumors showed a better survival rate. The DPD expression level of $\leq 7.49 \times 10^{-3}$ was associated with patient response and prolonged survival. The TP and ERCC1 expression levels were not associated with response and survival. The ERCC4 expression also had no correlation with response. However, patients with an expression level $\leq 11.24 \times 10^{-3}$ showed prolonged survival. The KU80 expression revealed no association with response. Nevertheless, patients with an expression level of $\leq 140.51$ $\times 10^{-3}$ showed a slightly prolonged survival. The GADD45A expression was weakly associated with clinical response and patients with an expression level of $\leq 82.18 \times 10^{-3}$ showed significantly increased survival. The combined expression of GADD $45 A$ and $T P$ was significantly correlated with survival. High expression of TP and/or GADD45A was associated with zero response and poor survival in patients with AGC treated with 5-FU and cisplatin-based preoperative chemotherapy.

The FOLFOX6 regimen, commonly used in preoperative chemotherapy, consists of oxaliplatin, calcium folinate, and 
5-FU. Hu et al. [34, 35] investigated the novel prognostic biomarkers for GC with FOLFOX6 preoperative chemotherapy in two different studies. One study showed that there was no association in the 15-PGDH expression in noncancerous GC tissues and GC tissues. However, the 15-PGDH expression in the preoperative chemotherapy group was higher than in the surgery alone group. Preoperative chemotherapy increased the 15-PGDH expression in GC patients. The elevated 15-PGDH expression was associated with better survival. The other study showed that intratumoral infiltration of Foxp3 Tregs was lower and that the dendritic cell density was higher in the preoperative chemotherapy group. This study demonstrated that preoperative chemotherapy reduced intratumoral Foxp3 Tregs infiltration while increasing dendritic cell density. Further, patients treated with preoperative chemotherapy manifested longer overall survival with lower infiltration of Foxp3 Tregs and higher dendritic cell density. Maskey et al. [36] also investigated the new prognostic biomarkers for GC with the same preoperative chemotherapy regimen: the expression of $\mathrm{B} 7-\mathrm{H} 4$ was significantly lower in preoperative chemotherapy group than in the surgery alone group. In the preoperative chemotherapy group, patients with low B7-H4 expression had longer overall survival. Above all, the level of $15-\mathrm{PGDH}$, infiltration of Foxp3 Tregs, dendritic cell density, and low B7-H4 expression served as useful prognostic biomarkers/predictors for ADC treated with FOLFOX6 regimen preoperatively. Qu et al. [37] conducted a clinical study to investigate whether the seven GC-related biomarkers served as predictors to preoperative chemotherapy. Using a preoperative chemotherapy regimen of mFOLFOX7, which was very similar to FOLFOX6, the expression of C-met, EGFR, HER2, Ki-67, MMP7, P53, and TOPII was tested in GC tissues before and after mFOLFOX7 preoperative chemotherapy. The response rate of patients with P53-negative expression was higher than that of P53positive expression. The efficacy of preoperative chemotherapy in HER2-positive patients was significantly higher than in HER2-negative patients. In summary, HER2 and P53 represent potential prognostic and efficacy predictors to treatment with preoperative mFOLFOX7 regimen. Bataille et al. [38] conducted a study investigating P53 expression in patients exposed to a preoperative chemotherapy regimen of etoposide, cisplatin, and mitomycin. They demonstrated that positive P53 immunostaining and P53 mutant status in tumors before preoperative chemotherapy were predictors of prognosis and treatment response.

Giampieri et al. [39] and Fareed et al. [40] conducted studies to investigate novel predictors in patients treated with preoperative platinum-based chemotherapy. The results of these studies demonstrated that mismatch repair deficiency, tumor regression, and ERCC1 nuclear protein expression predicted favorable prognosis in GC patients treated with preoperative platinum-based chemotherapy. Giampieri et al. studied metastatic GC while Fareed et al. researched locally advanced gastroesophageal cancer. Naka et al. [41] reported the value of organic cation transporter 2 (OCT2) expression in the prediction of response to cisplatin-based preoperative chemotherapy. The result was promising and suggested that increased expression of OCT2 predicted response to preoperative chemotherapy with S-1/cisplatin in GC.

Nevertheless, several studies discussed preoperative chemotherapy based on docetaxel.

Kubo et al. [42] and Okada et al. [43] investigated predictors of response to preoperative chemotherapy using combinations of docetaxel, 5-FU, and cisplatin. They found that BAK expression and overexpression of FoxMlin GC were potential prognostic markers in GC. Overexpression of FoxM1 also enhanced resistance to docetaxel. Schmitt et al. [44] showed that the transcriptional expression of eight genes predicted pathological response to docetaxel combined with trastuzumab-based preoperative chemotherapy.

Blank et al. [45] evaluated the prognostic and predictive value of two MTHFR gene polymorphisms in esophagogastric adenocarcinoma treated with preoperative chemotherapy. The MTHFP A1298C polymorphisms were associated with adverse outcome and served as an independent negative prognostic factor in GC treated with preoperative chemotherapy. Wu et al. [46] investigated the correlation of apoptotic and proliferation index with preoperative chemotherapy efficacy. A total of 167 patients were separated into 2 groups, one exposed to preoperative chemotherapy combined with surgery and another treated with surgery alone. In the preoperative chemotherapy group, the average apoptotic index (AI) was higher and AI/KI was significantly higher than that of surgery alone group, indicating that preoperative chemotherapy not only inhibited tumor proliferation but also promoted apoptosis. AI, KI, and AI/KI were associated with chemotherapy efficacy and prognosis in patients treated with preoperative chemotherapy. Jia et al. [47] investigated the role of death-associated protein-3 (DAP-3) in the evaluation of preoperative chemotherapy efficacy and prognosis of GC. The higher expression of DAP-3 was correlated with better prognosis.

A series of molecular mechanisms in the body may determine the efficacy of preoperative chemotherapy, suggesting new directions for precision medicine. Teng et al. [48] suggested that the Lin28/microRNA pathway might be a signaling pathway regulated by Lin 28 and was associated with resistance to chemotherapy in GC. Rubie et al. [49] suggested that the upregulation of CXCR4 mRNA was promoted by preoperative chemotherapy in GC patients. Higher tumor stages involving lymph and vein infiltration reduced CXCL12 mRNA expression. The regulation of a specific molecule alters the therapeutic precision, warranting the need for redefining preoperative chemotherapy.

In general, several studies investigated the potential predictors of response to different preoperative chemotherapy regimens. We believe that a combination of several potential predictors facilitates prediction of response to chemotherapy with precision (Table 3 ).

\section{Conclusions}

Resection is the ultimate goal of clinical intervention during the management of AGC to ensure longer progression-free survival. However, the 5-year survival rate of AGC patients undergoing R0 resection still remains poor. Preoperative 
TABLE 3: Genes or proteins related to response prediction in preoperative chemotherapy.

\begin{tabular}{|c|c|c|c|}
\hline Genes or proteins & Results & Regimens & Reference \\
\hline $\begin{array}{l}\text { TS, DPD, TP, ERCC1, } \\
\text { ERCC4, KU80, } \\
\text { GADD } 45 \text { A }\end{array}$ & $\begin{array}{l}\text { Tumor TS expression level of } \leq 344.19 \times 10^{-3} \text { improved } \\
\text { survival. } \\
\text { DPD expression level of } \leq 7.49 \times 10^{-3} \text { was associated with } \\
\text { patient response and prolonged survival. } \\
\text { Elevated expression of TP and/or GADD } 45 \text { A was associated } \\
\text { with zero response and poor survival. }\end{array}$ & 5-FU and cisplatin & Napieralski et al. [33] \\
\hline 15-PGDH & $\begin{array}{l}\text { High levels of } 15-\mathrm{PGDH} \text { expression were associated with } \\
\text { better survival. }\end{array}$ & FOLFOX6 & Hu et al. [34] \\
\hline $\begin{array}{l}\text { Foxp3 Tregs } \\
\text { dendritic cell }\end{array}$ & $\begin{array}{l}\text { Infiltration of Foxp3 Tregs and dendritic cells served as useful } \\
\text { prognostic biomarkers for AGC treated with FOLFOX6 } \\
\text { regimen preoperatively. }\end{array}$ & FOLFOX6 & Hu et al. [35] \\
\hline B7-H4 & $\begin{array}{l}\text { In the preoperative chemotherapy group, patients with low } \\
\text { B7-H4 expression had longer overall survival. }\end{array}$ & FOLFOX6 & Maskey et al. [36] \\
\hline HER2, P53 & $\begin{array}{l}\text { HER } 2 \text { and P53 were predictors of efficacy inmFOLFOX7 } \\
\text { preoperative chemotherapy. }\end{array}$ & mFOLFOX7 & Qu et al. [37] \\
\hline P53 & $\begin{array}{l}\text { Positive P53 immunostaining and P53 mutation in tumors } \\
\text { before preoperative chemotherapy might serve as molecular } \\
\text { predictors of response in patients with advanced GC treated } \\
\text { with preoperative chemotherapy. }\end{array}$ & $\begin{array}{l}\text { etoposide, cisplatin, } \\
\text { mitomycin }\end{array}$ & Bataille et al. [38] \\
\hline MMR & $\begin{array}{l}\text { Mismatch repair deficiency predicted favorable prognosis via } \\
\text { immune response activation in patients with metastatic GC } \\
\text { treated with preoperative platinum-based chemotherapy. }\end{array}$ & Platinum-based & Giampieri et al. [39] \\
\hline $\begin{array}{l}\text { Tumor regression } \\
\text { ERCC1 }\end{array}$ & $\begin{array}{l}\text { Tumor regression and ERCC1 nuclear protein expression are } \\
\text { promising predictive markers in gastroesophageal cancer } \\
\text { treated with preoperative platinum-based chemotherapy. }\end{array}$ & platinum-based & Fareed et al. [40] \\
\hline OCT2 & $\begin{array}{l}\text { High expression of OCT2 might represent a potential } \\
\text { predictor of response to preoperative chemotherapy with } \\
\text { S-1/cisplatin in GC. }\end{array}$ & $\begin{array}{l}\text { cisplatin-based (plus S-1 } \\
\text { or paclitaxel) }\end{array}$ & Naka et al. [41] \\
\hline BAK & $\begin{array}{l}\text { BAK expression in GC predicts chemotherapeutic response } \\
\text { and clinical prognosis in patients treated with preoperative } \\
\text { docetaxel chemotherapy. }\end{array}$ & $\begin{array}{l}\text { docetaxel, } 5-\mathrm{FU} \\
\quad \text { cisplatin }\end{array}$ & Kubo et al. [42] \\
\hline FoxM1 & $\begin{array}{l}\text { Overexpression of FoxM1 is a potential prognostic marker for } \\
\text { enhanced chemoresistance to docetaxel in GC. }\end{array}$ & $\begin{array}{l}\text { docetaxel, 5-FU, } \\
\text { cisplatin, } \mathrm{S}-1\end{array}$ & Okada et al. [43] \\
\hline 8 genes & $\begin{array}{l}\text { Transcriptional expression of } 8 \text { genes predicts pathological } \\
\text { response to docetaxel plus trastuzumab-based preoperative } \\
\text { chemotherapy }\end{array}$ & $\begin{array}{l}\text { docetaxel plus } \\
\text { trastuzumab }\end{array}$ & Schmitt et al. [44] \\
\hline MTHFP A1298C & $\begin{array}{l}\text { MTHFP A1298C polymorphisms were associated with poor } \\
\text { outcome and represent independent negative prognostic } \\
\text { factors in preoperative chemotherapy. }\end{array}$ & NS & Blank et al. [45] \\
\hline $\mathrm{AI}, \mathrm{KI}, \mathrm{AI} / \mathrm{KI}$ & $\begin{array}{l}\mathrm{AI}, \mathrm{KI} \text {, and } \mathrm{AI} / \mathrm{KI} \text { were associated with efficacy and prognosis } \\
\text { of patients in the preoperative chemotherapy group. }\end{array}$ & NS & Wu et al. [46] \\
\hline DAP-3 & $\begin{array}{l}\text { Higher expression of DAP- } 3 \text { was associated with better } \\
\text { prognosis in GC patients in the preoperative chemotherapy } \\
\text { group. }\end{array}$ & NS & Jia et al. [47] \\
\hline $\begin{array}{l}\text { Lin } 28 \\
\text { microRNA-107 }\end{array}$ & $\begin{array}{l}\text { Lin } 28 / \text { microRNA- } 107 \text { pathway was regulated by } \operatorname{Lin} 28 \text { in } \\
\text { possible GC chemoresistance. }\end{array}$ & $\begin{array}{l}\text { oxaliplatin, paclitaxel, } \\
\text { doxorubicin, and } \\
\text { fluorouracil }\end{array}$ & Teng et al. [48] \\
\hline $\begin{array}{l}\text { CXCL12 } \\
\text { CXCR4 }\end{array}$ & $\begin{array}{l}\text { CXCR4 mRNA upregulation following preoperative } \\
\text { chemotherapy in GC patients was directly related to response } \\
\text { and negatively correlated with higher tumor stages with } \\
\text { lymph and vein infiltration. }\end{array}$ & NS & Rubie et al. [49] \\
\hline
\end{tabular}

TS: thymidylate synthase; DPD: dihydropyrimidine dehydrogenase; TP: thymidine phosphorylase; ERCC1: excision repair cross complementing 1; ERCC4: excision repair cross complementing 4; KU80: an enzyme involved in nonhomologous end joining repair; GADD45A: growth arrest and DNA-damageinducible protein 45 alpha; 15-PGDH: 15-hydroxyprostaglandin dehydrogenase; DAP-3: death-associated protein-3; MMR: mismatch repair; OCT2: organic cation transporter 2; FoxM1: fork head box transcription factor 1; NS: not stated. 
chemotherapy is a clinical option with favorable results in terms of survival rate and overall outcomes in GC patients. Even though preoperative chemotherapy is beneficial for most AGC patients, a few patients still tend to manifest poor response and outcome. Rapid advances in the fields of genomics and proteomics have led to an era of precision medicine, which combines molecular targeting with preoperative chemotherapy to enhance the survival of AGC patients. However, larger and more robust clinical trials are needed to establish the value of combining perioperative chemotherapy with precision medicine.

\section{Competing Interests}

The authors of the manuscript hereby declare the absence of any conflict of interests.

\section{Acknowledgments}

This work was supported by two Grants from the National Natural Science Foundation of China (no. 30872477 and no. 30901729).

\section{References}

[1] J. Ferlay, I. Soerjomataram, R. Dikshit et al., "Cancer incidence and mortality worldwide: sources, methods and major patterns in GLOBOCAN 2012," International Journal of Cancer, vol. 136, no. 5, pp. E359-E386, 2015.

[2] L. Quéro, S. Guillerm, and C. Hennequin, "Neoadjuvant or adjuvant therapy for gastric cancer," World Journal of Gastrointestinal Oncology, vol. 7, no. 8, pp. 102-110, 2015.

[3] J. R. Siewert, K. Bottcher, J. D. Roder, R. Busch, P. Hermanek, and H. J. Meyer, "Prognostic relevance of systematic lymph node dissection in gastric carcinoma," British Journal of Surgery, vol. 80, no. 8, pp. 1015-1018, 1993.

[4] R. B. He and J.-Q. Chen, "Pre-operative chemotherapy for gastric cancer," The Journal of International Medical Research, vol. 37, no. 5, pp. 1259-1266, 2009.

[5] D. Cunningham, W. H. Allum, S. P. Stenning et al., "Perioperative chemotherapy versus surgery alone for resectable gastroesophageal cancer," New England Journal of Medicine, vol. 355, no. 1, pp. 11-20, 2006.

[6] J. A. Ajani, R. J. Mayer, D. M. Ota et al., "Preoperative and postoperative combination chemotherapy for potentially resectable gastric carcinoma," Journal of the National Cancer Institute, vol. 85, no. 22, pp. 1839-1844, 1993.

[7] K. Fujitani, J. A. Ajani, C. H. Crane et al., "Impact of induction chemotherapy and preoperative chemoradiotherapy on operative morbidity and mortality in patients with locoregional adenocarcinoma of the stomach or gastroesophageal junction," Annals of Surgical Oncology, vol. 14, no. 7, pp. 2010-2017, 2007.

[8] Y. Li and Z. Zhou, "Role and management of cancer clinical database in the application of gastric cancer precision medicine," Zhonghua Wei Chang Wai Ke Za Zhi, vol. 19, pp. 132137, 2016.

[9] I. Songun, H. J. Keizer, J. Hermans et al., "Chemotherapy for operable gastric cancer: results of the Dutch randomised FAMTX trial. The Dutch Gastric Cancer Group (DGCG)," European Journal of Cancer, vol. 35, no. 4, pp. 558-562, 1999.
[10] H. H. Hartgrink, C. J. H. van de Velde, H. Putter et al., "Neo-adjuvant chemotherapy for operable gastric cancer: long term results of the dutch randomised FAMTX trial," European Journal of Surgical Oncology, vol. 30, no. 6, pp. 643-649, 2004.

[11] M. Ychou, V. Boige, J.-P. Pignon et al., "Perioperative chemotherapy compared with surgery alone for resectable gastroesophageal adenocarcinoma: an FNCLCC and FFCD multicenter phase III trial," Journal of Clinical Oncology, vol. 29, no. 13, pp. 1715-1721, 2011.

[12] C. Schuhmacher, S. Gretschel, F. Lordick et al., "Neoadjuvant chemotherapy compared with surgery alone for locally advanced cancer of the stomach and cardia: European organisation for research and treatment of cancer randomized trial 40954," Journal of Clinical Oncology, vol. 28, no. 35, pp. 5210$5218,2010$.

[13] K. Oyama, S. Fushida, J. Kinoshita et al., "Efficacy of preoperative chemotherapy with docetaxel, cisplatin, and S-1 (DCS therapy) and curative resection for gastric cancer with pathologically positive para-aortic lymph nodes," Journal of Surgical Oncology, vol. 105, no. 6, pp. 535-541, 2012.

[14] J. Kinoshita, S. Fushida, T. Tsukada et al., "Efficacy of conversion gastrectomy following docetaxel, cisplatin, and S-1 therapy in potentially resectable stage IV gastric cancer," European Journal of Surgical Oncology, vol. 41, no. 10, pp. 1354-1360, 2015.

[15] D. Cunningham, N. Starling, S. Rao et al., "Capecitabine and oxaliplatin for advanced esophagogastric cancer," The New England Journal of Medicine, vol. 358, no. 1, pp. 36-46, 2008.

[16] Y.-K. Kang, W.-K. Kang, D.-B. Shin et al., "Capecitabine/ cisplatin versus 5 -fluorouracil/cisplatin as first-line therapy in patients with advanced gastric cancer: a randomised phase III noninferiority trial," Annals of Oncology, vol. 20, no. 4, pp. 666673, 2009.

[17] A. D. Roth, N. Fazio, R. Stupp et al., "Docetaxel, cisplatin, and fluorouracil; docetaxel and cisplatin; and epirubicin, cisplatin, and fluorouracil as systemic treatment for advanced gastric carcinoma: a randomized phase II trial of the Swiss group for clinical cancer research," Journal of Clinical Oncology, vol. 25, no. 22, pp. 3217-3223, 2007.

[18] M. Hirakawa, Y. Sato, H. Ohnuma et al., "A phase II study of neoadjuvant combination chemotherapy with docetaxel, cisplatin, and S-1 for locally advanced resectable gastric cancer: nucleotide excision repair (NER) as potential chemoresistance marker," Cancer Chemotherapy and Pharmacology, vol. 71, no. 3, pp. 789-797, 2013.

[19] E. Oki, Y. Emi, T. Kusumoto et al., "Phase II study of docetaxel and S-1 (DS) as neoadjuvant chemotherapy for clinical stage III resectable gastric cancer," Annals of Surgical Oncology, vol. 21, no. 7, pp. 2340-2346, 2014.

[20] J. S. Macdonald, S. R. Smalley, J. Benedetti et al., "Chemoradiotherapy after surgery compared with surgery alone for adenocarcinoma of the stomach or gastroesophageal junction," New England Journal of Medicine, vol. 345, no. 10, pp. 725-730, 2001.

[21] A. M. Lowy, B. W. Feig, N. Janjan et al., "A pilot study of preoperative chemoradiotherapy for resectable gastric cancer," Annals of Surgical Oncology, vol. 8, no. 6, pp. 519-524, 2001.

[22] J. Wydmański, R. Suwinski, S. Poltorak et al., "The tolerance and efficacy of preoperative chemoradiotherapy followed by gastrectomy in operable gastric cancer, a phase II study," Radiotherapy and Oncology, vol. 82, no. 2, pp. 132-136, 2007. 
[23] A. S. Allal, D. Zwahlen, M.-A. Bründler et al., "Neoadjuvant radiochemotherapy for locally advanced gastric cancer: longterm results of a phase I trial," International Journal of Radiation Oncology Biology Physics, vol. 63, no. 5, pp. 1286-1289, 2005.

[24] J. A. Ajani, P. F. Mansfield, N. Janjan et al., "Multi-institutional trial of preoperative chemoradiotherapy in patients with potentially resectable gastric carcinoma," Journal of Clinical Oncology, vol. 22, no. 14, pp. 2774-2780, 2004.

[25] J. A. Ajani, P. F. Mansfield, C. H. Crane et al., "Paclitaxel-based chemoradiotherapy in localized gastric carcinoma: degree of pathologic response and not clinical parameters dictated patient outcome," Journal of Clinical Oncology, vol. 23, no. 6, pp. 12371244, 2005.

[26] J. A. Ajani, K. Winter, G. S. Okawara et al., "Phase II trial of preoperative chemoradiation in patients with localized gastric adenocarcinoma (RTOG 9904): quality of combined modality therapy and pathologic response," Journal of Clinical Oncology, vol. 24, no. 24, pp. 3953-3958, 2006.

[27] M. Stahl, M. K. Walz, M. Stuschke et al., "Phase III comparison of preoperative chemotherapy compared with chemoradiotherapy in patients with locally advanced adenocarcinoma of the esophagogastric junction," Journal of Clinical Oncology, vol. 27, no. 6, pp. 851-856, 2009.

[28] E. Krasniqi, S. Pellicori, and V. Formica, "Emerging role of S1 in gastric cancer," Indian Journal of Medical and Paediatric Oncology, vol. 36, no. 4, pp. 219-228, 2015.

[29] T. Inoue, S. Yachida, H. Usuki et al., "Pilot feasibility study of neoadjuvant chemoradiotherapy with S-1 in patients with locally advanced gastric cancer featuring adjacent tissue invasion or JGCA bulky N2 lymph node metastases," Annals of Surgical Oncology, vol. 19, no. 9, pp. 2937-2945, 2012.

[30] D. J. Lee, T. S. Sohn, D. H. Lim et al., "Phase I study of neoadjuvant chemoradiotherapy with S-1 and oxaliplatin in patients with locally advanced gastric cancer," Cancer Chemotherapy and Pharmacology, vol. 69, no. 5, pp. 1333-1338, 2012.

[31] A. K. Trip, B. J. Poppema, M. I. Van Berge Henegouwen et al., "Preoperative chemoradiotherapy in locally advanced gastric cancer, a phase I/II feasibility and efficacy study," Radiotherapy and Oncology, vol. 112, no. 2, pp. 284-288, 2014.

[32] P. van Hagen, M. C. C. M. Hulshof, J. J. B. van Lanschot et al., "Preoperative chemoradiotherapy for esophageal or junctional cancer," The New England Journal of Medicine, vol. 366, no. 22, pp. 2074-2084, 2012.

[33] R. Napieralski, K. Ott, M. Kremer et al., "Combined GADD45A and thymidine phosphorylase expression levels predict response and survival of neoadjuvant-treated gastric cancer patients," Clinical Cancer Research, vol. 11, no. 8, pp. 3025-3031, 2005.

[34] M. Hu, K. Li, N. Maskey et al., "15-PGDH expression as a predictive factor response to neoadjuvant chemotherapy in advanced gastric cancer," International Journal of Clinical and Experimental Pathology, vol. 8, no. 6, pp. 6910-6918, 2015.

[35] M. Hu, K. Li, N. Maskey et al., "Decreased intratumoral Foxp3 Tregs and increased dendritic cell density by neoadjuvant chemotherapyassociated with favorable prognosis in advanced gastric cancer," International Journal of Clinical and Experimental Pathology, vol. 7, no. 8, pp. 4685-4694, 2014.

[36] N. Maskey, K. Li, M. Hu et al., "Impact of neoadjuvant chemotherapy on lymphocytes and co-inhibitory B7-H4 molecule in gastric cancer: low B7-H4 expression associates with favorable prognosis," Tumor Biology, vol. 35, no. 12, pp. 11837-11843, 2014.
[37] J.-J. Qu, Y.-R. Shi, and F.-Y. Hao, "Clinical study of the predictors to neoadjuvant chemotherapy in patients with advanced gastric cancer," Chinese Journal of Gastrointestinal Surgery, vol. 16, no. 3, pp. 276-280, 2013.

[38] F. Bataille, P. Rümmele, W. Dietmaier et al., "Alterations in p53 predict response to preoperative high dose chemotherapy in patients with gastric cancer," Molecular Pathology, vol. 56, no. 5, pp. 286-292, 2003.

[39] R. Giampieri, E. Maccaroni, A. Mandolesi et al., "Mismatch repair deficiency may affect clinical outcome through immune response activation in metastatic gastric cancer patients receiving first-line chemotherapy," Gastric Cancer, 2016.

[40] K. R. Fareed, A. Al-Attar, I. N. Soomro et al., "Tumour regression and ERCC1 nuclear protein expression predict clinical outcome in patients with gastro-oesophageal cancer treated with neoadjuvant chemotherapy," British Journal of Cancer, vol. 102, no. 11, pp. 1600-1607, 2010.

[41] A. Naka, R. Takeda, M. Shintani et al., "Organic cation transporter 2 for predicting cisplatin-based neoadjuvant chemotherapy response in gastric cancer," American Journal of Cancer Research, vol. 5, pp. 2285-2293, 2015.

[42] T. Kubo, Y. Kawano, N. Himuro et al., "BAK is a predictive and prognostic biomarker for the therapeutic effect of docetaxel treatment in patients with advanced gastric cancer," Gastric Cancer, vol. 19, no. 3, pp. 827-838, 2016.

[43] K. Okada, Y. Fujiwara, T. Takahashi et al., "Overexpression of forkhead box M1 transcription factor (FOXM1) is a potential prognostic marker and enhances chemoresistance for docetaxel in gastric cancer," Annals of Surgical Oncology, vol. 20, no. 3, pp. 1035-1043, 2013

[44] E. Schmitt, F. Végran, S. Chevrier et al., "Transcriptional expression of 8 genes predicts pathological response to first-line docetaxel + trastuzumab-based neoadjuvant chemotherapy," BMC Cancer, vol. 15, no. 1, article no. 169, 2015.

[45] S. Blank, S. Rachakonda, G. Keller et al., "A retrospective comparative exploratory study on two methylentetrahydrofolate reductase (MTHFR) polymorphisms in esophagogastric cancer: the A1298C MTHFR polymorphism is an independent prognostic factor only in neoadjuvantly treated gastric cancer patients," BMC Cancer, vol. 14, no. 1, article 58, 2014.

[46] A. Wu, Y. Jia, B. Dong et al., "Apoptosis and KI 67 index correlate with preoperative chemotherapy efficacy and better predict the survival of gastric cancer patients with combined therapy," Cancer Chemotherapy and Pharmacology, vol. 73, no. 5, pp. 885-893, 2014.

[47] Y. Jia, L. Ye, K. Ji et al., "Death-associated protein-3, DAP3 , correlates with preoperative chemotherapy effectiveness and prognosis of gastric cancer patients following perioperative chemotherapy and radical gastrectomy," British Journal of Cancer, vol. 110, no. 2, pp. 421-429, 2014.

[48] R. Teng, Y. Hu, J. Zhou et al., “Overexpression of Lin28 decreases the chemosensitivity of gastric cancer cells to oxaliplatin, paclitaxel, doxorubicin, and fluorouracil in part via microRNA107," PLoS ONE, vol. 10, no. 12, Article ID e0143716, 2015.

[49] C. Rubie, A. Kauffels, K. Kölsch, M. Glanemann, and C. Justinger, "CXCL12/CXCR4 display an inverse mRNA expression profile in gastric carcinoma that correlates with tumor progression," Oncology Letters, vol. 11, no. 1, pp. 360-364, 2016.

[50] I. Riquelme, K. Saavedra, J. A. Espinoza et al., "Molecular classification of gastric cancer: towards a pathwaydriven targeted therapy," Oncotarget, vol. 6, no. 28, pp. 24750-24779, 2015. 
[51] S. Lee and S. C. Oh, "Changing strategies for target therapy in gastric cancer," World Journal of Gastroenterology, vol. 22, no. 3, pp. 1179-1189, 2016.

[52] Y.-J. Bang, E. Van Cutsem, A. Feyereislova et al., "Trastuzumab in combination with chemotherapy versus chemotherapy alone for treatment of HER2-positive advanced gastric or gastrooesophageal junction cancer (ToGA): a phase 3, open-label, randomised controlled trial," The Lancet, vol. 376, no. 9742, pp. 687-697, 2010.

[53] K. Kataoka, M. Tokunaga, J. Mizusawa et al., "A randomized phase II trial of systemic chemotherapy with and without trastuzumab followed by surgery in HER2-positive advanced gastric or esophagogastric junction adenocarcinoma with extensive lymph node metastasis: Japan Clinical Oncology Group study JCOG1301 (Trigger Study)," Japanese Journal of Clinical Oncology, vol. 45, no. 11, pp. 1082-1086, 2015. 


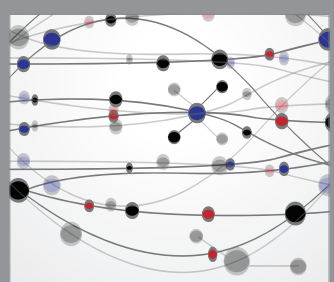

The Scientific World Journal
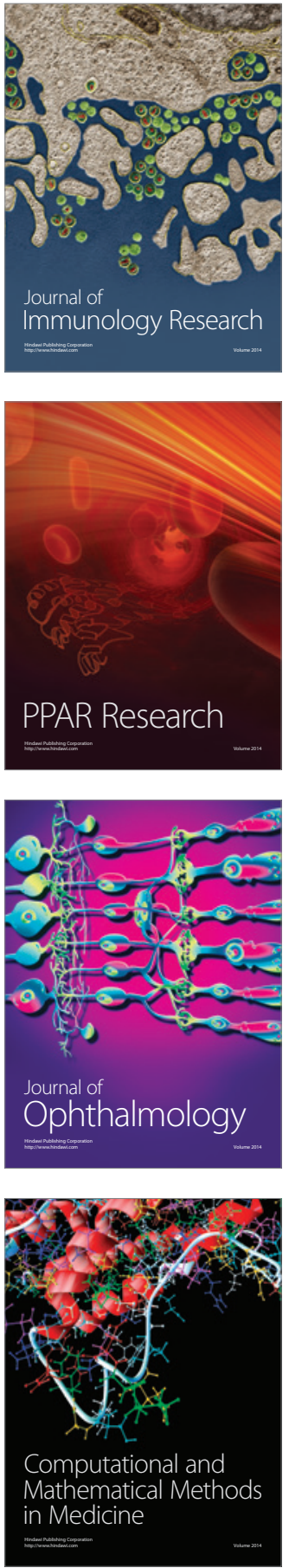

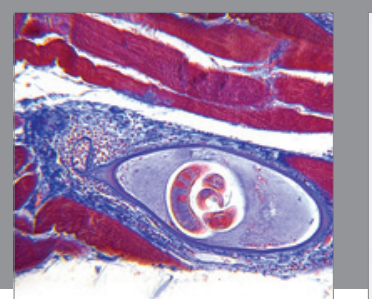

Gastroenterology Research and Practice

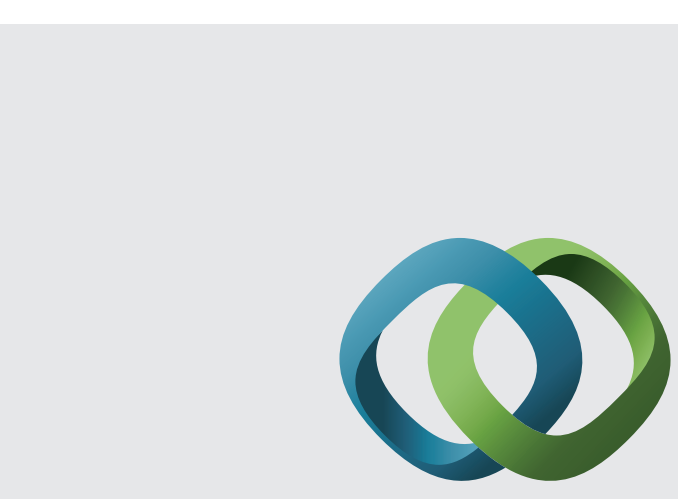

\section{Hindawi}

Submit your manuscripts at

http://www.hindawi.com
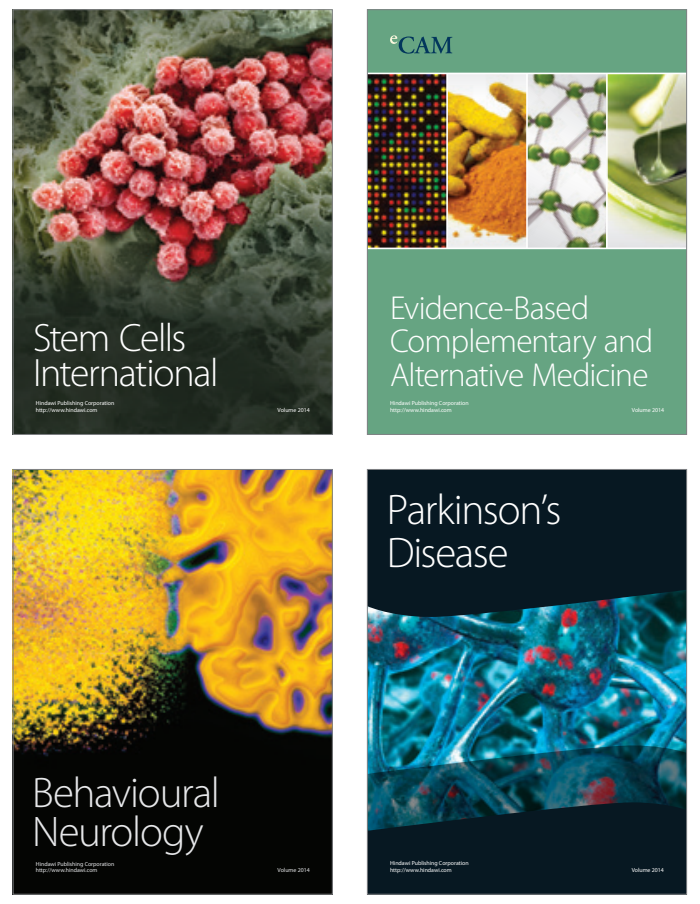
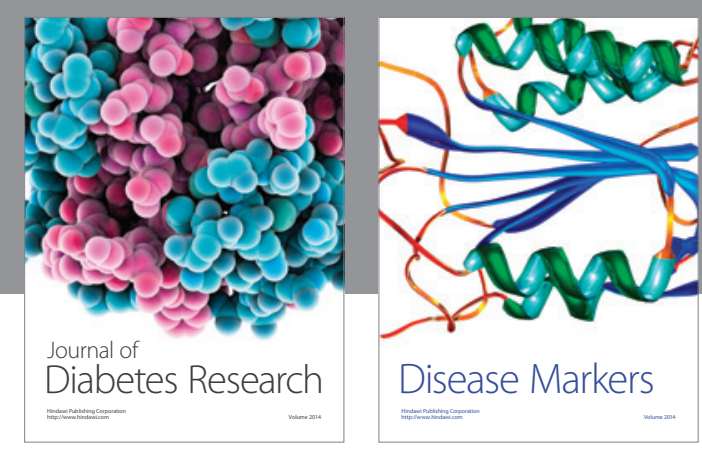

Disease Markers
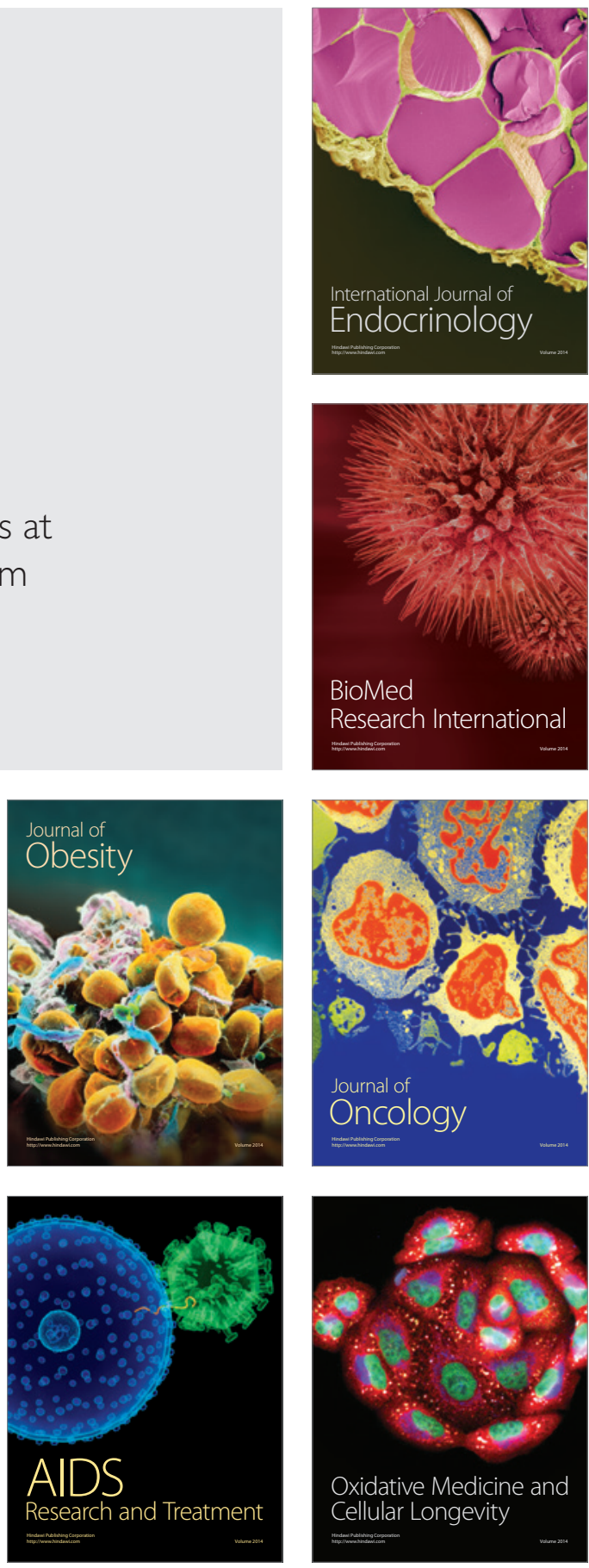